\section{Costo económico asociado a inactividad física en Chile}

\section{The economic burden of physical inactivity in Chile}

\section{Sr. Editor:}

La inactividad física se ha convertido en uno de los factores de riesgo más importante en el desarrollo de enfermedades crónicas no transmisibles (ECNTs) [1]. Ser inactivo (o realizar $<150 \mathrm{~min}$ de actividad física de intensidad moderada $0<75$ min de actividad física vigorosa a la semana), es causante de $9 \%$ de muertes prematuras en el mundo, equivalente a 5,3 millones de muerte por año ${ }^{1}$. Se estima que al reducir la inactividad física entre $10 \%$ a $25 \%$ a nivel mundial, el número de muertes prematuras se reduciría entre 0,5 y 1,3 millones por año ${ }^{1}$.

Actualmente un $31,1 \%$ de la población adulta a nivel mundial es físicamente inactiva, con prevalencias de inactividad que van desde $17 \%$ en el sureste Asiático, $43 \%$ en las Américas ${ }^{2}$ y $19,8 \%$ en la población chile$\mathrm{na}^{3}$. No obstante, hace muy poco se desconocía cual era el costo económico asociado a inactividad física.
Esta información es esencial para orientar la toma de decisiones en relación a definir políticas públicas y distribución del presupuesto nacional en áreas prioritarias y que podrían tener un alto impacto en el perfil epidemiológico del país. Es así como la segunda serie en actividad física y salud de la revista The Lancet, publicó en el año 2016 por primera vez el costo económico asociado a inactividad física a nivel mundial, incorporando información de 142 países (incluyendo Chile) que en su total representan el 93,2\% de la población en el mundo 4 .

Ding y cols, reportaron en este estudio que el costo total asociado a inactividad física en Chile durante el año 2013 fue de US\$ 103 millones de dólares al año, estimando que el costo directo fue de US\$ 69,2 millones, mientras que el costo indirecto correspondió a US $\$ 34,1$ millones, equivalente a un $0,23 \%$ del costo total asociado a la atención en salud (Total health-care cost) ${ }^{4}$. Este costo económico directo (US\$ 69,2 millones) asociado a inactividad física en Chile es cubierto en $47,4 \%$ por el sector público, $20,9 \%$ por el sector privado y $31,7 \%$ por el usuario. Del costo directo total asociado a inactividad física (US\$ 69,2 millones), US\$ 8,8 millones corresponden a gastos en enfermedad coronaria asociados a ser físicamente inactivo, US\$ 3,6 millones a enfermedad 
cerebrovascular, US $\$ 50,6$ millones a diabetes mellitus tipo 2, US\$ 3,0 millones a cáncer de mamas y US\$ 3,1 millones a cáncer de colon. Ding y cols, también reportaron que la perdida en productividad en Chile debido a mortalidad asociada a inactividad física fue equivalente a US\$ 34,1 millones en el año $2013^{4}$.

La importancia de practicar actividad física de forma regular y cumplir con las recomendaciones mínimas de actividad física tiene una alta relevancia al momento de disminuir el costo económico como también disminuir el riesgo asociado a ECNTs ${ }^{1,4}$. Ding y cols. estimaron que si la población físicamente inactiva en Chile cumpliera con las recomendaciones de actividad física el riesgo de ECNTs atribuible a inactividad física disminuiría en un 3,5\% para enfermedad coronaria, $4,0 \%$ para enfermedad cerebrovascular, $4,4 \%$ para diabetes mellitus tipo 2, 6,5\% para cáncer de mamas, 6,3\% para cáncer de colon y 5,7\% para mortalidad general ${ }^{4}$.

Estas evidencias entregadas por Ding y cols., son muy relevantes en el ámbito de la salud pública, pudiendo servir como base para generar conciencia a nivel gubernamental de lo esencial que es la implementación de políticas orientadas a la promoción de estilos de vida saludable y, principalmente, políticas orientadas a incrementar la práctica de actividad física en la población chilena. Los antecedentes presentados demuestran que la adopción de hábitos en actividad física juega un rol esencial en la disminuición del costo económico como también así en la disminuición del riesgo de ECNTs en la población. Políticas públicas que se orienten a fomentar la práctica regular de actividad física en todos los grupos etarios son esenciales, partiendo por incrementar las horas de actividad física en los establecimientos educacionales, como también así implementar la prescripción de actividad física en pacientes que asistan a centros de atención primaria de salud, implementación de áreas verdes y vías de transporte público que promuevan la práctica de actividad física al aire libre y actividad física asociada al transporte ${ }^{5,6}$, medidas que podrían ser esenciales claves para incrementar los niveles de actividad física de la población.

\footnotetext{
Carlos Celis-Morales ${ }^{1,2}$, Carlos Salas ${ }^{3}$, María Adela Martínez $z^{4}$ Ana María Leiva Alex Garrido-Méndez ${ }^{6}$, Ximena Díaz-Martínez $z^{7}$ ${ }^{1}$ BHF Glasgow Cardiovascular Research Centre, Institute of Cardiovascular and Medical Science, University of Glasgow, Glasgow, United Kingdom. ${ }^{2}$ Centro de Fisiología y Biomecánica, Universidad Mayor, Santiago, Chile. ${ }^{3}$ Departamento de Educación Física, Facultad de Educación, Universidad de Concepción. Concepción, Chile.
}

\author{
${ }_{4}^{4}$ Instituto de Farmacia, Facultad de Ciencias, \\ Universidad Austral de Chile, Valdivia, Chile. \\ ${ }^{5}$ Instituto de Anatomía, Histología y Patología, \\ Facultad de Medicina, Universidad Austral de Chile, \\ Valdivia, Chile. \\ ${ }^{6}$ Escuela de Educación Física, Universidad San \\ Sebastián, Concepción, Chile. \\ ${ }^{7}$ Grupo de Investigación Calidad de Vida, \\ Departamento de Educación, Universidad del Bío-Bío, \\ Chillan, Chile.
}

\section{Referencias}

1. Lee IM, Shiroma EJ, Lobelo F, Puska P, Blair SN, Katzmarzyk PT, et al. Effect of physical inactivity on major non-communicable diseases worldwide: an analysis of burden of disease and life expectancy. Lancet 2012; 380 (9838): 219-29. doi: 10.1016/s0140-6736(12)61031-9.

2. Hallal PC, Andersen LB, Bull FC, Guthold R, Haskell W, Ekelund U. Global physical activity levels: surveillance progress, pitfalls, and prospects. Lancet 2012; 380 (9838): 247-57. doi: 10.1016/S0140-6736(12)60646-1

3. MINSAL. Encuesta Nacional de Salud 2009-2010. Chile: Ministerio de Salud, 2010. Available: http://web.minsal.cl/ portal/url/item/bcb03d7bc28b64dfe040010165012d23.pdf

4. Ding D, Lawson KD, Kolbe-Alexander TL, Finkelstein EA, Katzmarzyk PT, van Mechelen W, et al. The economic burden of physical inactivity: a global analysis of major non-communicable diseases. The Lancet 2016; 388 (10051): 1311-24. doi: 10.1016/S0140-6736(16)30383-X

5. Celis-Morales CA, Lyall DM, Welsh P, Anderson J, Steell L, Guo Y, et al. Association between active commuting and incident cardiovascular disease, cancer, and mortality: prospective cohort study. BMJ 2017; 357 (4): j1456-64. doi: https://doi.org/10.1136/bmj.j1456.

6. Lear SA, Hu W, Rangarajan S, Gasevic D, Leong D, Iqbal $\mathrm{R}$, et al. The effect of physical activity on mortality and cardiovascular disease in 130,000 people from 17 high-income, middle-income, and low-income countries: the PURE study. The Lancet 2017; 9 (17): 1-12. doi: 10.1016/S01406736(17)31634-3.

Correspondencia a: Dr Carlos Celis Morales BHF Glasgow Cardiovascular Research Centre Institute of Cardiovascular \& Medical Sciences University of Glasgow 126 University Place. Glasgow G12 8TA. United Kingdom. Carlos.Celis@glasgow.ac.uk 